\title{
Treatment strategy for multisegmental cervicomedullary ependymoma: illustrative case
}

\author{
Andrei A. Zrelov, MD, PhD, ${ }^{1}$ Malik M. Tastanbekov, MD, PhD, ${ }^{1}$ Mikhail V. Alexandrov, MD, PhD, ${ }^{2}$ Anastasiia S. Nechaeva, MD, ${ }^{1}$ \\ Olga A. Toporkova, MD, ${ }^{2}$ Olga M. Vorobeva, MD, PhD, ${ }^{3}$ and Konstantin A. Samochernykh, MD, $\mathrm{PhD}^{1}$
} Departments of ${ }^{1}$ Fourth Neurosurgery, ${ }^{2}$ Clinical Neurophysiology, and ${ }^{3}$ Pathomorphology, Polenov Neurosurgical Research Institute, branch of the Almazov National Medical
Research Center, St. Petersburg, Russia

\begin{abstract}
BACKGROUND Cervicomedullary ependymoma (CME) is a rare tumor of the central nervous system. The CME treatment strategy is insufficiently represented in the literature and is a complex task for neurosurgeons.

OBSERVATIONS The authors describe an infrequent case of a large multisegmental CME that extended from the medulla oblongata to the cervical spinal cord at the level of the sixth cervical vertebra in a 21-year-old female. Neurological disorders presented with headache, dysphagia, hypophonia, and weakness in the limbs. Subtotal removal of the tumor was performed according to intraoperative neurophysiological monitoring (IONM) results. A wait-and-see approach with patient follow-up was chosen.

LESSONS Total tumor removal of the CME is the most important favorable prognostic factor. Subtotal resection can be considered if the borders of the tumor are unclear and the result of IONM is unfavorable. The role of postoperative radiation therapy in the case of subtotal removal of the tumor remains controversial.
\end{abstract}

https://thejns.org/doi/abs/10.3171/CASE21608

KEYWORDS ependymoma; intramedullary; cervicomedullary; medulla oblongata; cervical spinal cord

Ependymomas account for approximately 2-9\% of all tumors of the central nervous system. ${ }^{1-4}$ Literature sources on the surgical treatment of cervicomedullary ependymoma (CME) are extremely limited. In the available literature, only 2 studies are mentioned that present a series of clinical observations of patients with $\mathrm{CME}{ }^{2,4}$ The prevalence of CME is relatively low: $3.8-10.0 \%$ of all spinal cord ependymomas and up to $23.7 \%$ of all cervicomedullary tumors. ${ }^{5-7}$ CME constitutes a complex surgical pathology, especially in patients with multisegmental distribution in the spinal cord.

Aggressive surgery for these neoplasms remains controversial due to the high risk of neurological disorders, including respiratory disorders, dysphagia, motor deficits, and even tetraplegia. Currently, due to the rapid development of neuroimaging techniques and various intraoperative technologies, such as intraoperative neurophysiological monitoring (IONM), the treatment of these neoplasms has become more radical with better long-term results. ${ }^{2,4,8}$ However, due to insufficient coverage of CME in the literature, there is no unified, standardized treatment strategy for these patients. In this report, we present a rare case of multisegmental CME in a woman in her early 20 s and describe the features of the clinical characteristics, diagnostics, surgical treatment with IONM, and prognostic factors in patients with CME.

\section{Illustrative Case}

A 21 -year-old patient presented to the 4th Neurosurgery Department of the Polenov Neurosurgical Institute with headache, bulbar symptoms, and weakness in the limbs. The first clinical manifestation was pain in the cervical and thoracic spine. Acute deterioration had occurred in the past month. Neurological examination revealed weakness in the extremities (2/5 on the right upper limb proximal,

ABBREVIATIONS CME = cervicomedullary ependymoma; IONM = intraoperative neurophysiological monitoring; MRI = magnetic resonance imaging; SSEP = somatosensory evoked potentials.

INCLUDE WHEN CITING Published December 20, 2021; DOI: 10.3171/CASE21608.

SUBMITTED October 22, 2021. ACCEPTED November 8, 2021.

(c) 2021 The authors, CC BY-NC-ND 4.0 (http://creativecommons.org/licenses/by-nc-nd/4.0/). 
$2+/ 5$ on the left upper limb proximal, $3 / 5$ on the upper limbs distal, $3+/ 5$ on the lower extremities proximal, and $4 / 5$ on the lower extremities distal according to the Oxford Scale of muscle testing), numbness in the hands, positional vertigo, unsteadiness on walking, decreased visual acuity, and bulbar symptoms such as dysphagia and hypophonia (3 points on the modified McCormick Scale).

Magnetic resonance imaging (MRI) of the cervical spine showed an intramedullary cervicomedullary cystic solid tumor. It extended from the medulla oblongata to the $\mathrm{C} 6$ vertebral level. The size of the solid component was $82 \times 18 \times 11 \mathrm{~mm}$, and the cystic component was $36 \times 33 \times 33 \mathrm{~mm}$ (Fig. $1 \mathrm{~A}-\mathrm{C})$.

The patient underwent a C1-6 laminotomy and microsurgical removal of the intramedullary tumor with IONM. First, we performed fenestration of the cystic mass. Next, we created several midline incisions along the posterior surface of the spinal cord to preserve the large arterial supply. A gray-white tumor of soft elastic consistency with clear borders and moderate blood supply was visualized. The lesion was dissected and subtotally removed (Fig. 2A-D).

Polymodal IONM was performed to monitor the functional state of the spinal cord pathways. Transcranial electrical stimulation and D-wave registration were performed to assess the safety of the descending motor pathways. Before manipulations, stable motorevoked responses were recorded from the distal target muscles of the arms, and unstable motor-evoked responses were recorded from the proximal target muscles of the hands and target muscles of the legs. To assess the integrity of the ascending pathways of the spinal cord, somatosensory evoked potentials (SSEP) were monitored during electrical stimulation of the median nerves of both arms and the tibial nerves of both legs. ${ }^{9}$

Transient disappearance of motor-evoked responses from the right biceps brachii muscle, right brachioradialis muscle, and right thenar (Fig. 3) were registered during the surgery. For the purpose of correcting the perfusion of the spinal cord, the surgery was suspended for 10 minutes, during which the spinal cord was irrigated with a warm saline solution and the anesthesiology team temporarily maintained blood pressure $10 \mathrm{~mm} \mathrm{Hg}$ above the target. As a result of these measures, stable motor responses from the target muscles of the right arm were restored in 10 minutes. The D-wave amplitude varied within $20 \%$ during the surgery, indicating a satisfactory functional state of the descending pathways of the spinal cord.

At the end of the surgery, when we removed the rostral fragment of the tumor during transcranial stimulation, the amplitude of motor responses from the target muscles of the right arm decreased to $40 \%$ of the "base value" (before the surgery). There was a steady decrease in the SSEP amplitude of $70-80 \%$ upon stimulation with the left median nerve, right median nerve, left tibial nerve, and right tibial nerve. The D-wave amplitude decreased to $50 \%$ of the initial level. The registered changes in neurophysiological parameters went beyond the acceptable risk range, which is why the neurosurgeon decided to stop removing the tumor.

Postoperative T1-weighted spinal MRI confirmed subtotal resection and did not reveal any surgical complications (Fig. 4).

Postoperatively, the patient showed worsened motor weakness of the right hand (1/5 on the right upper extremity proximal) and decreased sensation in both hands. However, on the 5th day after the surgery, the muscle strength in the right arm corresponded to the preoperative level, bulbar disorders became less common, and the patient was discharged.

The pathological examination revealed an ependymoma, grade II. The level of Ki-67 proliferative activity was $1-3 \%$ (Fig. $5 A-D)$. A wait-and-see approach with patient follow-up was chosen.

\section{Discussion}

\section{Observations}

CME is a rare surgical pathology. Most often, CME occurs in the fourth decade of life, with a ratio of men to women of $0.65-1.00 /$ 1.00 . $^{2,4}$ For tumors with the predominant component located in the medulla oblongata, the clinical picture of the disease is usually characterized by dysfunction of the lower group of cranial nerves with motor and sensory deficits. In cases of predominant spread of the tumor along the cervical spinal cord, symptoms include signs of cervical myelopathy. ${ }^{2,4,5}$ Local neck pain is also very common, and hypertension symptoms are less common. The patient in this case
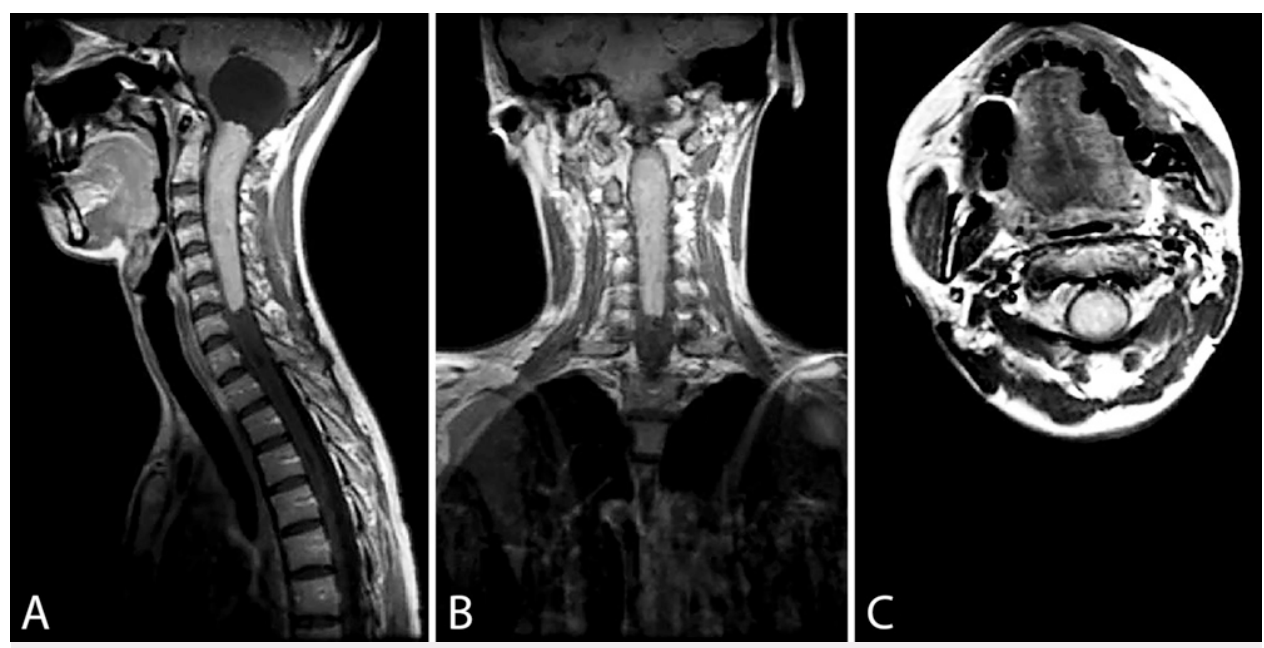

FIG. 1. MRI of the cervicothoracic spine with posterior cranial fossa before the surgery (T1-weighted with contrast enhancement). The solid component showed an intense enhancement on gadolinium. A: Sagittal view. B: Coronal view. C: Axial view. 


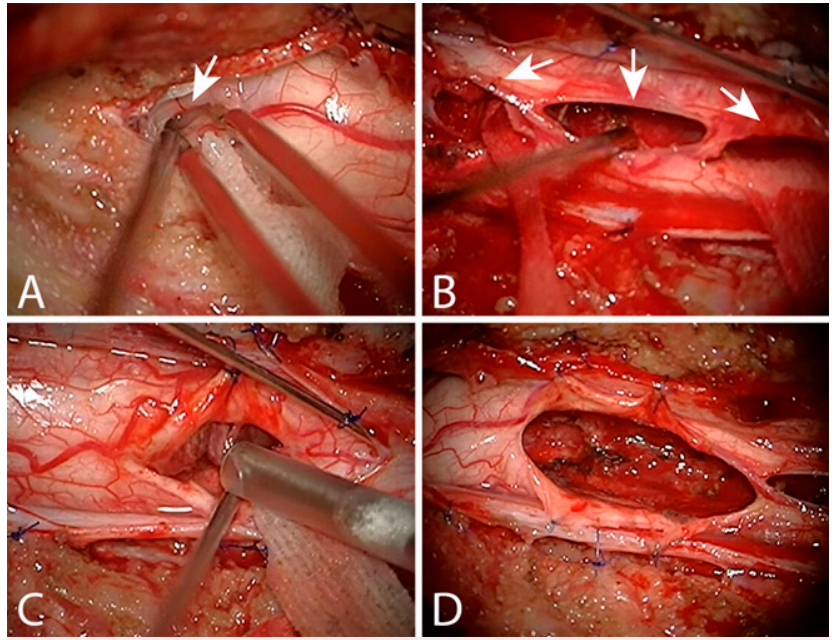

FIG. 2. Intraoperative images. A: Dissection of the cystic component of the tumor in the medulla oblongata (arrow). B: Several midline incisions were performed (arrows). C: Use of an ultrasonic disintegrator to remove the tumor. D: Final view of the rostral part of the surgical wound after removal of the tumor.

had significant neurological deficits, spinal fragments of the tumor prevailed, and motor disorders of the peripheral type in the proximal parts of the arms dominated.

In most cases, CMEs are benign slow-growing tumors, and patients have a long history of the disease. ${ }^{10}$ In a large series of observations by $\mathrm{Ge}$ et al. ${ }^{2}$ and Li et al.,, the duration of symptoms prior to diagnosis was 1-72 months, with an average duration of

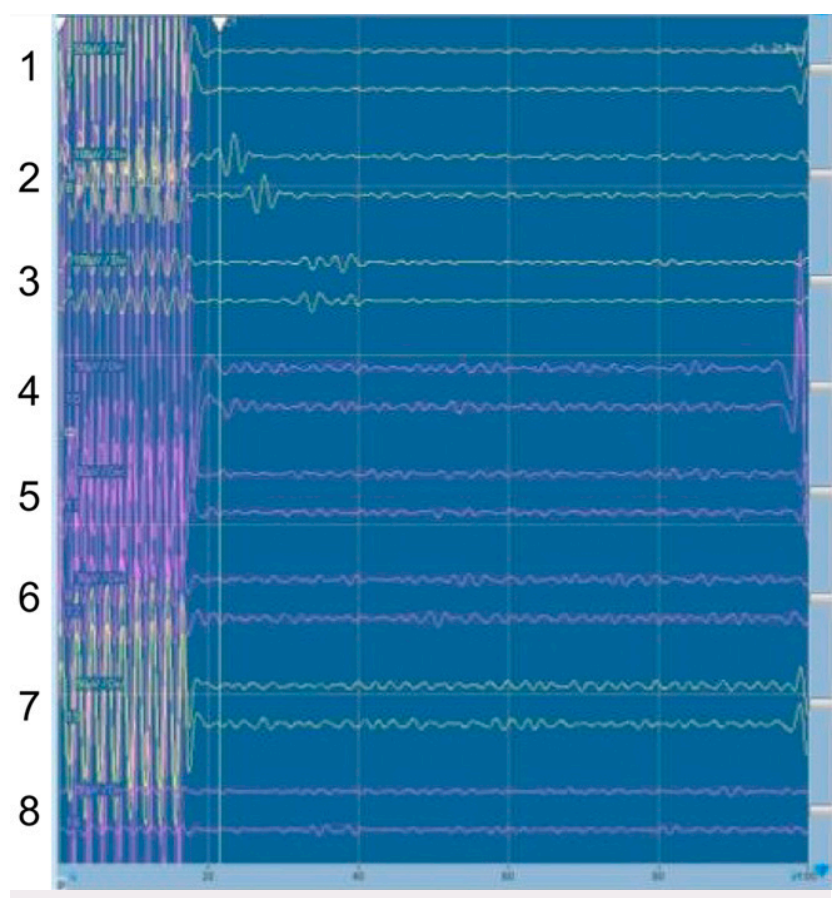

FIG. 3. Multipulse transcranial stimulation: M-responses from the left arm muscles (tracks 1, 2, and 3). The M-responses from the muscles of the right arm (tracks 4, 5, and 6), left leg (7), and right leg (8) were not recorded. The left part of the panel is a stimulation artifact.
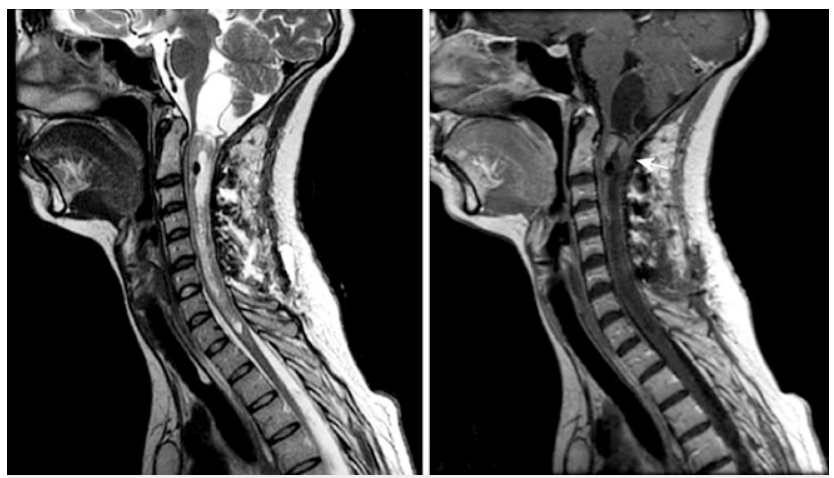

FIG. 4. MRI of the cervicothoracic spine with posterior cranial fossa after surgery. Zone of accumulation of contrast material along the upper pole in the zone of postoperative changes (level C1 of the vertebra) measuring $7 \times 5 \times 10 \mathrm{~mm}$. Left: T2-weighted sagittal view.

Right: T1-weighted sagittal view with contrast enhancement.

14.2-20.1 months, which reflects the slow growth of these tumors. In our case, the duration of the disease history was approximately 12 months. Acute occurrence or increase in symptoms that could indicate a hemorrhage in the tumor is not typical for CME. However, Kutty et al. ${ }^{5}$ described a clinical case of a patient with an acute onset of the disease in the form of sudden dysesthesia in the arms and legs in which MRI of the brain and cervical spinal cord showed a solid cystic CME with signs of hemorrhage.

The main treatment for CME is surgical removal of the tumor. Several studies have shown that total removal of these tumors is the most significant factor associated with a favorable prognosis of the disease. ${ }^{2,4,5}$ In the case of a high risk of neurological deficit with dense adhesion of the tumor to the brain and with significant deterioration in IONM parameters, subtotal removal of the tumor is preferable to prevent the increase in neurological symptoms in the

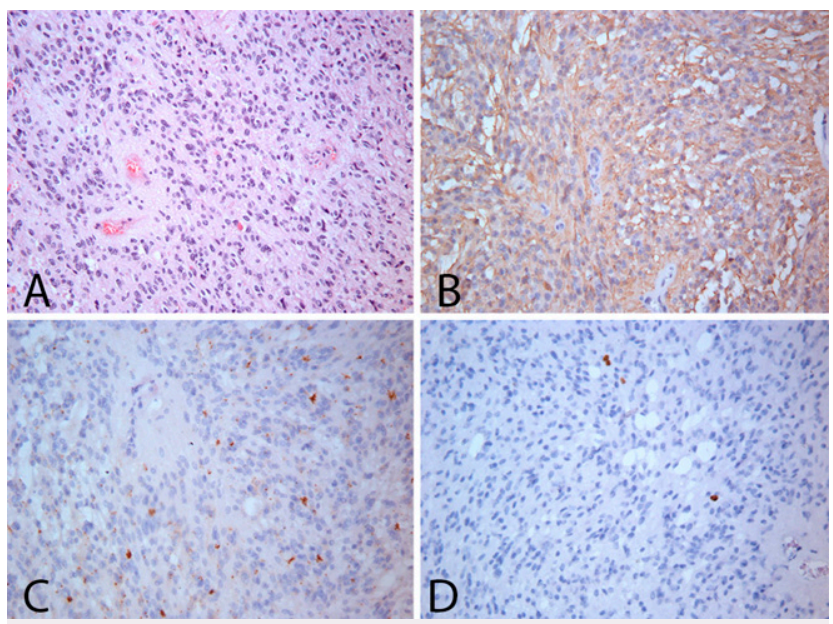

FIG. 5. Histopathological examination shows grade II ependymoma. A: Tumor cells with round nuclei and a "salt and pepper" type chromatin distribution with the formation of intense perivascular pseudorosettes. Staining with hematoxylin and eosin, original magnification $\times 200$. B: Glial fibrillary acidic protein cytoplasmic staining, original magnification $\times 200$. C: Spot staining of the epithelial membrane antigen in the rosette area, original magnification $\times 200$. D: Low proliferative activity shown by Ki-67, original magnification $\times 200$. 
postoperative period. ${ }^{2,5,11}$ In our case, there was an intense adhesion of the rostral fragment of the tumor more to the right. During removal of this part of the tumor, a significant decrease in the amplitude of the D-wave and SSEP were observed; therefore, the decision was made to carry out subtotal removal of the tumor.

The optimal time for performing surgery is controversial, especially for patients with small CMEs who have no neurological deficit at the preoperative level or have only local pain in the cervical spine (type 1 according to the modified McCormick Scale). ${ }^{10}$ According to a number of studies, it is better to perform the surgery as early as possible. ${ }^{4,12}$

One can predict the opportunity for total removal of the tumor using preoperative MRI data. A clear border between the tumor and the brain must meet at least one of the following conditions: the presence of a clear subarachnoid space between the tumor and healthy spinal cord tissue according to T2-weighted MRI scans and the smooth border between the tumor and the brain according to T1-weighted images with contrast enhancement. ${ }^{2}$ There is no need for direct surgical intervention for syringomyelic cysts that often accompany $\mathrm{CME}^{2,13}$

In the early postoperative course, $9-67 \%$ of patients may experience a deterioration in their neurological status. ${ }^{14-16}$ The need for tracheotomy was reported in $10.7-28.9 \%$ of cases, and the need for a nasogastric tube was reported in $21.4 \%{ }^{2,4}$ These manipulations were temporary in all cases. ${ }^{2,4}$

In our clinical case, there were positive changes after the surgery in the form of a decrease in the severity of dysphagia compared with the preoperative level, and there were no respiratory disorders.

Total removal of the tumor results in the best possible outcome of treatment. $2,17,18$ The size of the spinal tumor fragment may be a significant prognostic factor. Patients with a larger tumor may have a poorer outcome. ${ }^{19}$ In most cases, the spinal fragment of the CME extends 1-2 cervical segments. ${ }^{2,4}$ In the present case, CME extended to the level of the 6th cervical vertebral body, which is extremely rare and significantly complicates the surgery.

A number of authors consider it necessary to conduct radiation therapy in the postoperative period for subtotal removal of the tumor. ${ }^{2,20}$ However, according to other researchers, adjuvant radiation therapy does not improve overall patient survival. ${ }^{18}$ On the basis of our observation, taking into account the histological image (grade II) and the location of the residual tumor fragment, we preferred a wait-and-see approach with dynamic patient observation.

\section{Lessons}

CMEs are rare central nervous system tumors. The most important favorable prognostic factor is total tumor removal, which is potentially achievable in most cases due to the clear boundaries of the tumor with surrounding tissues. Subtotal resection can be considered if the borders of the tumor are unclear and the result of IONM is unfavorable. The role of postoperative radiation therapy in the case of subtotal removal of the tumor, especially in CME grade I/II, remains controversial.

\section{Acknowledgments}

We acknowledge support from Almazov National Medical Research Center for OpenAccess Publishing.

\section{References}

1. Cappelletti M, Ruggeri AG, lacopino G, Delfini R. Giant cell ependymoma of cervicomedullary junction: a case report of a long-term survivor and literature review. World Neurosurg. 2018;116:121-126.

2. Ge X, Wu Z, Zhang J, Zhang L. Surgical strategies and functional outcome of intramedullary cervicomedullary ependymoma. Turk Neurosurg. 2017;27(4):563-572.

3. Guyotat J, Metellus P, Giorgi R, et al. Infratentorial ependymomas: prognostic factors and outcome analysis in a multi-center retrospective series of 106 adult patients. Acta Neurochir (Wien). 2009; 151(8):947-960.

4. Li D, Hao SY, Wu Z, Jia GJ, Zhang LW, Zhang JT. Intramedullary medullocervical ependymoma-surgical treatment, functional recovery, and long-term outcome. Neurol Med Chir (Tokyo). 2013;53(10): 663-675.

5. Kutty RK, Ohmori K, Yamada Y, Kato Y. Cervicomedullary ependymoma with hemorrhage: a case report and review of literature. Asian J Neurosurg. 2020;15(1):190-193.

6. Oh MC, Kim JM, Kaur G, et al. Prognosis by tumor location in adults with spinal ependymomas. J Neurosurg Spine. 2013;18(3): 226-235.

7. Safaee M, Oh MC, Mummaneni PV, et al. Surgical outcomes in spinal cord ependymomas and the importance of extent of resection in children and young adults. J Neurosurg Pediatr. 2014;13(4): 393-399.

8. Sun J, Wang Z, Li Z, Liu B. Microsurgical treatment and functional outcomes of multi-segment intramedullary spinal cord tumors. J Clin Neurosci. 2009;16(5):666-671.

9. Simon MV, Borges L. Intramedullary spinal cord tumor resection. In: Simon MV, ed. Intraoperative Neurophysiology: A Comprehensive Guide to Monitoring and Mapping. Springer; 2018:389-423.

10. Aghakhani N, David P, Parker F, Lacroix C, Benoudiba F, Tadie M. Intramedullary spinal ependymomas: analysis of a consecutive series of 82 adult cases with particular attention to patients with no preoperative neurological deficit. Neurosurgery. 2008;62(6): 1279-1286.

11. Klekamp J. Spinal ependymomas. Part 1: Intramedullary ependymomas. Neurosurg Focus. 2015;39(2):E6.

12. Eroes CA, Zausinger S, Kreth FW, Goldbrunner R, Tonn JC. Intramedullary low grade astrocytoma and ependymoma. Surgical results and predicting factors for clinical outcome. Acta Neurochir (Wien). 2010;152(4):611-618.

13. Deng $X$, Wang $K$, Wu L, et al. Intraspinal hemangioblastomas: analysis of 92 cases in a single institution: clinical article. J Neurosurg Spine. 2014;21(2):260-269.

14. Chen P, Sui M, Ye J, Wan Z, Chen F, Luo C. An integrative analysis of treatment, outcomes and prognostic factors for primary spinal anaplastic ependymomas. J Clin Neurosci. 2015;22(6):976-980.

15. Schebesch KM, Mueller S, Wendl C, Brawanski A, Riemenschneider MJ, Proescholdt M. Recurrence rates and functional outcome after resection of intrinsic intramedullary tumors. Clin Neurol Neurosurg. 2015;134:60-66.

16. Yuce I, Sade R, Calõkoglu C, Ogul H, Kantarci M. Anaplastic ependymoma of spinal cord presented with low back pain. Spine $J$. 2015;15(8):1894.

17. Rodríguez D, Cheung MC, Housri N, Quinones-Hinojosa A, Camphausen K, Koniaris LG. Outcomes of malignant CNS ependymomas: an examination of 2408 cases through the Surveillance, Epidemiology, and End Results (SEER) database (1973-2005). J Surg Res. 2009;156(2):340-351.

18. Lin Y, Smith ZA, Wong AP, Melkonian S, Harris DA, Lam S. Predictors of survival in patients with spinal ependymoma. Neurol Res. 2015;37(7):650-655. 
19. Li TY, Chu JS, Xu YL, et al. Surgical strategies and outcomes of spinal ependymomas of different lengths: analysis of 210 patients: clinical article. J Neurosurg Spine. 2014;21(2):249-259.

20. Kasper EM, Ippen FM, Maragkos GA, Anderson MP, Rojas R, Mahadevan A. Tanycytic ependymoma of the brain stem, presentations of rare cystic disease variants and review of literature. J Neurosurg Sci. 2018;62(1):78-88.

\section{Disclosures}

The authors report no conflict of interest concerning the materials or methods used in this study or the findings specified in this paper.

\section{Author Contributions}

Conception and design: Zrelov, Tastanbekov, Alexandrov,

Samochernykh. Acquisition of data: Zrelov, Nechaeva, Toporkova,
Vorobeva. Analysis and interpretation of data: Zrelov, Tastanbekov, Alexandrov, Nechaeva. Drafting the article: Zrelov, Nechaeva,

Toporkova, Vorobeva. Critically revising the article: Zrelov, Tastanbekov, Alexandrov. Reviewed submitted version of manuscript: Samochernykh. Approved the final version of the manuscript on behalf of all authors: Zrelov. Administrative/technical/material support: Samochernykh.

\section{Correspondence}

Andrei A. Zrelov: Polenov Neurosurgical Research Institute, branch of the Almazov National Medical Research Center, St. Petersburg, Russia. andrey.zrelov89@gmail.com. 